\title{
Smooth Autonomous Navigation Considering Avoidance Behavior Characteristics of Pedestrians
}

Ryunosuke Harada ( $\nabla$ ryunosuke-harada222@g.ecc.u-tokyo.ac.jp )

University of Tokyo - Kashiwa Campus: Tokyo Daigaku - Kashiwa Campus https://orcid.org/00000003-1069-9110

Hiroshi Yoshitake

University of Tokyo - Kashiwa Campus: Tokyo Daigaku - Kashiwa Campus

Motoki Shino

University of Tokyo - Kashiwa Campus: Tokyo Daigaku - Kashiwa Campus

\section{Research Article}

Keywords: Autonomous navigation, Pedestrian avoidance, Personal mobility vehicle, Smooth traffic

Posted Date: January 12th, 2022

DOI: https://doi.org/10.21203/rs.3.rs-1215934/v1

License: (c) (1) This work is licensed under a Creative Commons Attribution 4.0 International License. Read Full License 


\section{Abstract}

To ensure the coexistence of autonomous personal mobility vehicles (PMVs) and pedestrians in a pedestrian zone, they should be able to smoothly pass across and avoid each other. Studies suggest that it is possible that PMVs and pedestrians can pass each other in a short period of time without compromising their comfort; this can be achieved through understanding how pedestrians react to the behavior of PMVs and by modifying the autonomous navigation of PMVs accordingly. Therefore, in this study, the avoidance behavior characteristics of pedestrians were investigated. Experiments were conducted to understand the influence of the selected avoiding behavior parameters and to understand the behavior characteristics of pedestrians in relation to the behavior of PMVs. Furthermore, a path planning strategy that enables smooth passing was developed based on these characteristics. The usefulness of this method was evaluated. The avoidance time and the avoiding angular velocity at the start and end of the avoidance behavior were the parameters that contributed to smooth autonomous navigation. The results show that pedestrian tolerance improves and the avoidance width decreases depending on these parameters. Furthermore, smooth autonomous navigation can be achieved using the characteristics of pedestrians' cognition against PMVs.

\section{Introduction}

Aging is a global phenomenon. Particularly in Japan, with the declining birthrate and the increase in the aging population, a number of social problems have arisen. These problems include an increase in the percentage of traffic accidents involving older drivers and a shortage of labor in the transportation industry. Personal mobility vehicles (PMVs) and service robots moving autonomously are attracting attention as solutions to these social problems [1, 2]. In the future, autonomous vehicles are expected to move in a pedestrian zone where they will coexist with pedestrians.

These autonomous vehicles should be safe from collisions and should be able to pass pedestrians smoothly. The strategy most commonly used in the path planning for autonomous mobility is to completely give way to pedestrians in order to avoid collisions, but considering that pedestrians also see mobility vehicles and avoid them to some extent, there should be a better strategy to achieve smooth passing. In previous studies, a number of methods have been explored for smooth passing. Liu et al. developed a method that incorporates the concept of personal space, which is the range in which pedestrians cannot tolerate the presence of others [3]. It is known that this personal space depends on the pedestrian density and the size of the moving object $[4,5]$. Peter et al. succeeded in developing a robotic algorithm that selects a safer and shorter path in a crowded situation than those taken by actual pedestrians and existing state-of-the-art path planning algorithms. It mimics the behavior of humans passing each other in a harmonious manner and creates a path even within a narrow avoidance area [6].

In order to achieve smooth passing, we focused on the "active strategy" method, in which the mobility vehicle starts to avoid the pedestrian before the pedestrian starts avoidance [7]. Avoidance behavior characteristics, such as how pedestrians perceive, judge, and act based on the avoidance behavior of the 
mobility vehicle in a passing situation, are important in formulating this strategy. Therefore, the purpose of this research is to develop a smooth autonomous navigation system that considers the avoidance behavioral characteristics of pedestrians for PMVs.

First, we selected the target parameter of avoidance behavior from the reference studies. Then, we identified the behavioral characteristics of pedestrians through an experiment using an actual PMV. Finally, a smooth autonomous navigation strategy was developed, and its usefulness was evaluated.

\section{Hypothesis Of Parameters Affecting Smoothness}

In this study, the classification of the smoothness of passing behavior was divided into two elements: shortening the avoidance time and not impairing comfort. With respect to the avoidance time, the goal was to reduce the avoidance width when passing pedestrians, which is an important factor in choosing a short path, even for small areas. On the other hand, a route with a short travel time may be selected, but it will not be acceptable if the vehicle behaves in an inadmissible maneuver to the surrounding pedestrians. Therefore, the goal of the navigation strategy developed in this study is to reduce the avoidance width when passing pedestrians without choosing a path that is unacceptable.

We focused on the avoidance behavior of the vehicle as a parameter that contributes to the improvement of smoothness. To select the target avoidance behavior of PMVs, a pedestrian behavior model consisting of three processes (cognition, judgment, and action) was adopted by referring to existing studies [8]. In this model, pedestrians recognize the behavior of a vehicle, predict the possibility of collision, judge the appropriateness of the side spacing and acceptability of the vehicle's behavior, and behave according to the avoidance width that was set as a target.

In a previous study, the following parameters were found to affect the subjective evaluation of pedestrians: moving velocity, avoidance starting distance, offset interval distance, turning angular velocity, and avoidance width [9]. Based on the pedestrian behavior model, the turning angular velocity and the avoiding width were selected from a previous study to be the parameters that more significantly affect pedestrian behavior and contribute to avoidance smoothness.

The hypothesis is that when the avoiding width of the vehicle is small, pedestrians perceive that the PMV is within their personal space and therefore judge the behavior of the PMV to be inappropriate and unacceptable. As a result, the pedestrian may increase the avoidance width owing to the anxiety of a possible collision. On the other hand, when the turning angular velocity is small, pedestrians are less likely to recognize the behavior of the vehicle, which reduces predictability and, consequently, validity. As a result, pedestrians may increase their avoidance width because of the anxiety caused by the lack of predictability.

\section{Investigation Of Avoidance Behavior Characteristics Of Pedestrians}




\subsection{Methods}

To understand the influence of avoidance behavior parameters on pedestrians, an experiment was conducted using an actual vehicle. Twenty men and women in their twenties participated in the experiment (A-T). The participants were instructed to walk towards a target point located $12 \mathrm{~m}$ away at a constant speed, and to pass a vehicle coming from the target point. The vehicle also moved at a constant speed $\left(V_{R}=0.7 \mathrm{~m} / \mathrm{s}\right)$ and started its avoidance behavior when the distance between the vehicle and the pedestrian falls below $7.2 \mathrm{~m}$. This distance was measured in a preliminary experiment as the distance required for the vehicle to start avoiding pedestrians before they start avoidance. The positional relationship between the pedestrian and the vehicle was calculated using the k-means method by measuring point cloud data from two LiDAR sensors (VLP-16, Velodyne Lidar, Inc.). The visual behavior of the pedestrians was measured using an eye-tracking device (Tobii Pro Glasses 2, Tobii AB). After each trial, the participants evaluated the PMV's avoidance behavior using a questionnaire. The participants rated their tolerance for the vehicle behavior on a 7-point Likert scale, with 1-3 being inadmissible, 4 being neutral, and 5-7 being admissible. Their introspection was also surveyed using a think-aloud protocol.

The experiment was conducted under different conditions to determine the avoidance width and the turning angular velocity, the two parameters that were selected in the previous chapter.

\subsection{Conditions}

In this experiment, for a certain avoiding time $T$, the vehicle turns at an angular velocity of $+\omega_{t}$ up to $T / 2$, and turns at $-\omega_{t}$ from $T / 2$ to $T$. For the avoidance behavior defined in this way, the vehicle's avoiding width, $y_{\text {veh }}$, is determined by equation (1).

$$
y_{v e h}\left(T, \omega_{t}\right)=\frac{2 V_{R}}{\omega_{t}}\left(1-\cos \omega_{t} T\right)
$$

1

In this experiment, one of the avoidance behavior parameters was fixed, and the other parameter was changed by varying the avoidance behavior time $T$. The six patterns of avoidance behavior shown in Table 1 were reproduced and tested. In the four conditions from $p-1$ to $p-4$, the turning angular velocity was fixed, and the behavior characteristics of the pedestrian due to the difference in the avoiding width of the vehicle were determined. In the three conditions $p-3, p-5$, and $p-6$, the avoiding width was fixed, and the change in behavior characteristics due to the difference in the turning angular velocity was determined. 
Table 1

Conditions of scenes in the characterization experiment

\begin{tabular}{|llll|}
\hline Condition ID & $\begin{array}{l}\text { Avoiding time } \\
T[\mathbf{s}]\end{array}$ & $\begin{array}{l}\text { Angular velocity } \\
\boldsymbol{\omega}_{t}[\mathrm{rad} / \mathrm{s}]\end{array}$ & $\begin{array}{l}\text { Avoiding width } \\
\boldsymbol{y}_{\text {ped }}[\mathrm{m}]\end{array}$ \\
\hline $\mathrm{p}-1$ & 4.0 & 0.25 & 0.69 \\
\hline $\mathrm{p}-2$ & 3.0 & 0.25 & 0.39 \\
\hline $\mathrm{p}-3$ & 2.0 & 0.25 & 0.17 \\
\hline $\mathrm{p}-4$ & 1.0 & 0.25 & 0.04 \\
\hline $\mathrm{p}-5$ & 3.0 & 0.11 & 0.17 \\
\hline $\mathrm{p}-6$ & 4.0 & 0.06 & 0.17 \\
\hline
\end{tabular}

\subsection{Results}

Figures 1 and 2 show the results of the avoidance width and tolerance evaluation, respectively, when the avoiding distance of the vehicle is changed. The pedestrian avoidance width increased as the vehicle's avoiding distance decreased, and the total width showed a decreasing trend. Based on the concept of personal space, the total avoidance width should not decrease. This result suggests that pedestrians use information other than the relative distance to the PMV as a factor in their decisions. This supports the hypothesis of the previous chapter. In the tolerance evaluation, participants became less admissible as the avoiding width of the PMV decreased. The result of the Kruskal-Wallis test revealed that there was a significant effect of avoidance width on tolerance evaluation $(p<0.01)$. In particular, the distribution of the tolerance evaluation changed significantly between $\mathrm{p}-2$ and $\mathrm{p}-3$, i.e., when the avoidance behavior time $T$ decreased from $3 \mathrm{~s}$ to $2 \mathrm{~s}$. As shown in Figures 1 and 2, a trade-off between avoidance width and tolerance was observed. These results suggest that the avoiding width of the PMV is not a suitable parameter for improving the smoothness of avoidance.

Figures 3 and 4 show the results of the avoidance width and tolerance evaluation, respectively, when the turning angular velocity of the vehicle is changed. The results showed that the avoidance width was almost constant regardless of the turning angular velocity. This rejects the hypothesis of the previous chapter. In the tolerance evaluation, participants became more admissible as the turning angular velocity decreased. The number of respondents who answered "inadmissible" was the lowest in the p- 5 condition, and it increased for both large- and small-angular-velocity conditions. Similar to the effect of avoidance width, there was a significant effect of turning angular velocity on tolerance evaluation $(p<0.01)$. In particular, there was a significant difference in the distribution of tolerance evaluation between $p-5$ and $p$ 3 , i.e., when the avoidance behavior time $T$ decreased from 3 seconds to 2 seconds.

\subsection{Discussion}


From the above results, there was differences in pedestrian's avoidance width and tolerance evaluation depending on the PMV's avoidance behavior. Thus, the change in pedestrian's observation and recognition in accordance with the PMV's behavior and how these changes affect their maneuver and introspection is discussed. Pedestrians' observations were analyzed using the results of their eye tracking data during the experiment, and their recognition through their comments after each condition.

\subsubsection{Gaze characteristics of pedestrians}

From the results, there was a significant difference in tolerance evaluation between the conditions where the PMV's avoidance time was 2 and 3 seconds. Thus, the behavioral changes of pedestrians between these conditions were investigated. The results of the gaze measurement showed that the participants stopped gazing at the PMV between 2 and $3 \mathrm{~s}$, as shown in Figure 5. In conditions where the PMV's avoiding time was less than two seconds, the gazing time was longer than the avoiding time. In contrast, the gazing time was shorter than the avoiding time in the condition of more than three seconds. This means that the pedestrian didn't gaze at the end of PMV's avoiding behavior only when the PMV's avoiding time was more than three seconds. In addition, when pedestrians perceive the end of the PMV's avoiding behavior, their gaze time and avoidance width increase, and their tolerance decreases (Figure 6). Therefore, to increase the smoothness of passing, the completion of the PMV avoidance is considered to be better after the pedestrian has stopped gazing at the PMV.

\subsubsection{Classification of avoidance maneuver}

Among the comments from the participants who rated the vehicle's behavior as unacceptable, many mentioned that the vehicle was slow to start its avoidance behavior. However, as mentioned in Section 3.1, the starting time of the avoidance behavior was set to a fixed value. These comments were more common in conditions with slower angular velocity. On the other hand, conditions with a higher angular velocity received comments indicating discomfort because they felt like the PMV was coming closer to them at the final stages of the PMV's avoidance behavior.

These two types of comments suggest that the impact of the turning angular velocity on pedestrians differs depending on the timing of their observations. Therefore, pedestrian avoidance maneuvers were classified, and the effect of the turning angular velocity was analyzed for each of them.

An example of the trajectory of the pedestrian avoidance maneuver is shown in Figure 7. There are two major points at which the pedestrian maneuver changes in their avoidance. The first is the "avoidance starting point," which is the result of the pedestrian's judgment on how to avoid the vehicle after recognizing the start of its avoidance behavior. The second is the "tipping point," which is thought to be the result of the pedestrian's judgment of the route to their final destination after averting their gaze away from the vehicle's behavior. In this experiment, the representative point of the pedestrian was scattered due to the pedestrian's gait in the range of $\pm 0.05 \mathrm{~m}$. Therefore, these two behavior changing points were measured considering the gait. 
First, the relationship between the pedestrian's avoidance starting point and the vehicle's turning angular velocity was compared. It was found that the smaller the angular velocity of the vehicle was, the slower the pedestrian started avoiding based on the start of the PMV's avoidance (Figure 8). This suggests that pedestrians delay making decisions and taking actions because of the reduced ease in recognizing the PMV's avoidance initiation.

The pedestrians' behavior after the tipping point was divided into two types: going straight on and turning to their destination. In the conditions where the effect of angular velocity was compared ( $p-3, p-5, p-6)$, $46 \%$ of the participants went straight while the others changed their direction. Among them, those who rated the vehicle's movement as inadmissible were more likely to go straight $(71 \%)$. This tendency was more pronounced when the angular velocity of the vehicle was higher. In condition $p-3$, where the angular velocity was the fastest, $100 \%$ of the subjects went straight when they rated the vehicle's movement as inadmissible. Comments also suggest that the decrease in pedestrian tolerance at the end of the vehicle's avoidance behavior was due to vehicle's large angular velocity, leading to an easier recognition of the PMV's avoidance termination. Since choosing to go straight leads to an increase in avoidance width, it can also be suggested that low tolerance, especially at the end of the vehicle's avoidance behavior, has a negative effect on the pedestrian's avoidance width.

\section{Design Of Smooth Autonomous Navigation Strategy}

The behavioral characteristics identified in the experiments highlighted the importance of designing the PMV's movements so that the start of the avoidance behavior is easily recognized and the end of avoidance behavior is not. Turning angular velocity at the start of the behavior, $\omega_{a}$, contributes to the recognition of the start of avoidance behavior. Turning angular velocity at the end of the behavior, $\omega_{b}$, and the avoiding behavior time, $T$, contribute to the recognition of the end of avoidance behavior. Thus a path planning strategy to adjust these three parameters was designed, as shown in Figure 9.

In the proposed strategy, $y_{\text {veh }}$ is determined by Equation (2) based on the geometric relationship shown in Figure 9. The constraint in Equation (3) is required to maintain the same posture angle at the beginning and end of the avoidance behavior.

$$
y_{v e h}\left(T, T_{r}, \omega_{a}, \omega_{b}\right)=\frac{V_{R}}{\omega_{a}}\left(1-\cos \omega_{a} T_{r}\right)+\frac{V_{R}}{\omega_{b}}\left\{1-\cos \omega_{b}\left(T-T_{r}\right)\right\}
$$

2

$$
\omega_{a} T_{r}=\omega_{b}\left(T-T_{r}\right)
$$

3

The requirement for this smooth autonomous navigation strategy is to be able to adjust the avoidance behavior time and turning angular velocity at the start and end of the avoidance behavior. The avoiding 
behavior time can be adjusted by changing the parameter $T$. The turning angular velocities can be adjusted by adjusting the timing of reversing the turning angular velocity which can be done by changing the parameter $T_{r}$.

\section{Evaluation Of Smooth Autonomous Navigation Strategy 5.1. Methods}

To evaluate whether the designed path planning strategy described in the previous section can improve the smoothness of passing pedestrians, an experiment was conducted in the same environment as described in Chapter 3. An actual device was used to implement the proposed strategy. Twenty men and women in their twenties participated in this experiment $(\mathrm{A} 2-\mathrm{T} 2)$.

The experimental conditions were as follows: the avoiding width of the PMV was fixed at $0.17 \mathrm{~m}$, and the avoiding time was set to $2 \mathrm{~s}$ or $3 \mathrm{~s}$, as shown in Table 2 . In addition, by changing the ratio of the time before and after the reversal of the turning angular velocity over a constant avoidance time, the degree of the turning angular velocity was adjusted. These different conditions were compared and evaluated to determine whether they affected the pedestrian's avoidance width and tolerance.

Table 2

Conditions of each scenario in the evaluation experiment

\begin{tabular}{|llll|}
\hline Condition ID & $\begin{array}{l}\text { Avoiding time } \\
T[s]\end{array}$ & $\begin{array}{l}\text { Time allocation ratio } \\
T_{\dot{r}} T-T_{r}\end{array}$ & $\omega_{a,} \omega_{b[\mathrm{rad} / \mathrm{s}]}$ \\
\hline q-1 & 2.0 & $1: 1$ & $0.250,0.250$ \\
\hline q-2 & 2.0 & $1: 2$ & $0.375,0.188$ \\
\hline q-3 & 2.0 & $1: 3$ & $0.500,0.167$ \\
\hline q-4 & 3.0 & $1: 1$ & $0.111,0.111$ \\
\hline q-5 & 3.0 & $1: 2$ & $0.116,0.083$ \\
\hline q-6 & 3.0 & $1: 3$ & $0.221,0.074$ \\
\hline
\end{tabular}

\subsection{Results}

The results for the avoidance width are shown in Figure 10. Comparing the avoidance times of $2 \mathrm{~s}$ and 3 $\mathrm{s}$, the avoidance width was reduced in the $3 \mathrm{~s}$ condition when compared to the $2 \mathrm{~s}$ condition. As for the ratio of the vehicle's turning angular velocity, the larger the angular velocity was in the first half and the smaller it was in the second half, the smaller the pedestrian's avoidance width tended to be. This angular velocity ratio also showed a positive effect on pedestrian tolerance (Figure 11). 
There were comments from the participants such as "I felt that I wanted the vehicle to avoid more in the other avoidance conditions, but in q- 5 and q- 6 I felt comfortable to avoid more," "I felt that it showed me the direction it wanted me to go," and "It guided me well." These comments suggests that the pedestrians had observed behaviors that affect the improvement of smoothness.

In summary, by adjusting the parameters of the avoiding behavior of the PMV in the designed path planning strategy, the pedestrian's tolerance improved and the avoidance width was reduced. Therefore, it was found that the proposed path planning strategy has a positive influence on smoothness in a pedestrian zone where pedestrians and autonomous vehicles coexist.

\section{Conclusions}

The purpose of this study was to develop a smooth autonomous navigation strategy for autonomous PMV that can suppress the avoidance width of pedestrians in passing situations without compromising their tolerance by allowing the autonomous vehicle to adopt an active strategy that accounts for the avoidance behavior characteristics of pedestrians. Experiments were conducted to understand the influence of the selected avoiding behavior parameters and to understand the behavior characteristics of pedestrians in relation to the behavior of PMV.

As a result, it was found that in terms of smooth passing, it is better if the start of the avoidance behavior of the vehicle is easily recognized by pedestrians and the end of the avoiding behavior is not. The experiment also showed that the turning angular velocity at the start of the behavior contributes to the pedestrian's recognition of the start of the avoidance behavior, and that the turning angular velocity at the end of the behavior as well as the avoiding behavior time contribute to the recognition of the end of the behavior.

Based on these results, a path planning strategy for smooth autonomous navigation was proposed and evaluated. In this strategy, the avoidance time and the turning angular velocity at the start and end of the avoidance behavior were adjustable because they were the parameters that contributed to smooth passing. The results show that pedestrian tolerance improves and the avoidance width is reduced by adjusting the parameter in the proposed path planning strategy. This indicates that the parameters were conducive to improving the smoothness of passing between PMV and pedestrians.

There were a few limitations of this study. While there are multiple pedestrians in a pedestrian zone where PMVs are expected to be introduced, this experiment only examined the effect on a single pedestrian. Therefore, it is necessary to additionally verify whether the characteristics obtained in this study are also valid for the pedestrian group. Moreover, experiments with wider range of age and a larger sample size are necessary to generalize the findings of this study.

\section{Declarations}

\section{Availability of data and materials}


Please contact the authors for data requests.

\section{Ethics approval and consent to participate}

The experimental procedures and other information were explained to the participants before the experiment, and informed consent was obtained. The experiment was conducted with the approval of the Ethics Committee of the University of Tokyo.

\section{Consent for publication}

I give my consent for information to be published in ROBOMECH journal.

\section{Competing interests}

The authors declare that they have no competing interests.

\section{Funding}

This study was funded by a project (JPNP18014) commissioned by the New Energy and Industrial Technology Development Organization.

\section{Authors' contributions}

All authors designed the study concept. RH conducted the experiments, analyzed the data, and wrote the manuscript. All authors read and approved the final manuscript.

\section{Acknowledgements}

This work was supported by a project commissioned by the New Energy and Industrial Technology Development Organization (NEDO). We would like to thank Editage (www.editage.com) for English language editing.

\section{Authors' information}

Department of Human and Engineered Environmental Studies, Graduate School of Frontier Sciences, The University of Tokyo, 5-1-5 Kashiwanoha, Kashiwa, Chiba, Japan

\section{References}

1. Ministry of Land, Infrastructure, Transport and Tourism (2012) Guidelines for the Introduction of Micro-Mobility. https://www.mlit.go.jp/common/000212867.pdf Accessed 26 Dec 2021 (in Japanese)

2. Advanced Information and Telecommunications Network Society (IT strategic headquarters) (2020) ITS public-private initiatives/road map. 
http://www.kantei.go.jp/jp/singi/it2/kettei/pdf/20200715/2020_roadmap.pdf Accessed 26 Dec 2021 (in Japanese). Prime Minister's Office of Japan

3. Liu C, Ooeda Y, Sumi T (2008) A model for pedestrian movement with obstacle evasion using personal space concept (in Japanese). Doboku Gakkai Ronbunshuu D 64:513-524. https://doi.org/10.2208/jscejd.64.513

4. Pham TQ, Nakagawa C, Shintani A, Ito T (2015) Evaluation of the effects of a personal mobility vehicle on multiple pedestrians using personal space. IEEE Transport Intell Transport Syst 16:20282037. https://doi.org/10.1109/TITS.2014.2388219

5. Nakagawa C, Imamura K, Shintani A, Ito T (2012) Experimental study on the influence of the size of personal mobility vehicle on pedestrians. Trans Jpn Soc Mech Eng C 78:3332-3342

6. Trautman P, Krause A (2010) Unfreezing the robot: Navigation in dense, interacting crowds The 2010 IEEE/RSJ International Conference on Intelligent Robots and Systems

7. Miyamoto K, Yoshioka H, Watanabe N, Takefuji Y (2014) Modeling of cooperative behavior agent based on collision avoidance decision process. The 28th Annual Conference of the Japanese Society for Artificial Intelligence

8. Mori H, Kurahashi T, Iwase T, Machida T, Kozato A, Yamashita M, Kitaoka H (2009) Modeling of pedestrian behavior for evaluating safety systems. Information Processing Society of Japan 50:3175-3185

9. Yoda M, Shiota Y (2000) Mobile robot's passing motion algorithm based on subjective evaluation. Trans Jpn Soc Mech Eng C (in Japanese) 66:3380-3387

\section{Figures}


घ Total width $\quad$ Pedestrian avoidance width

1.4

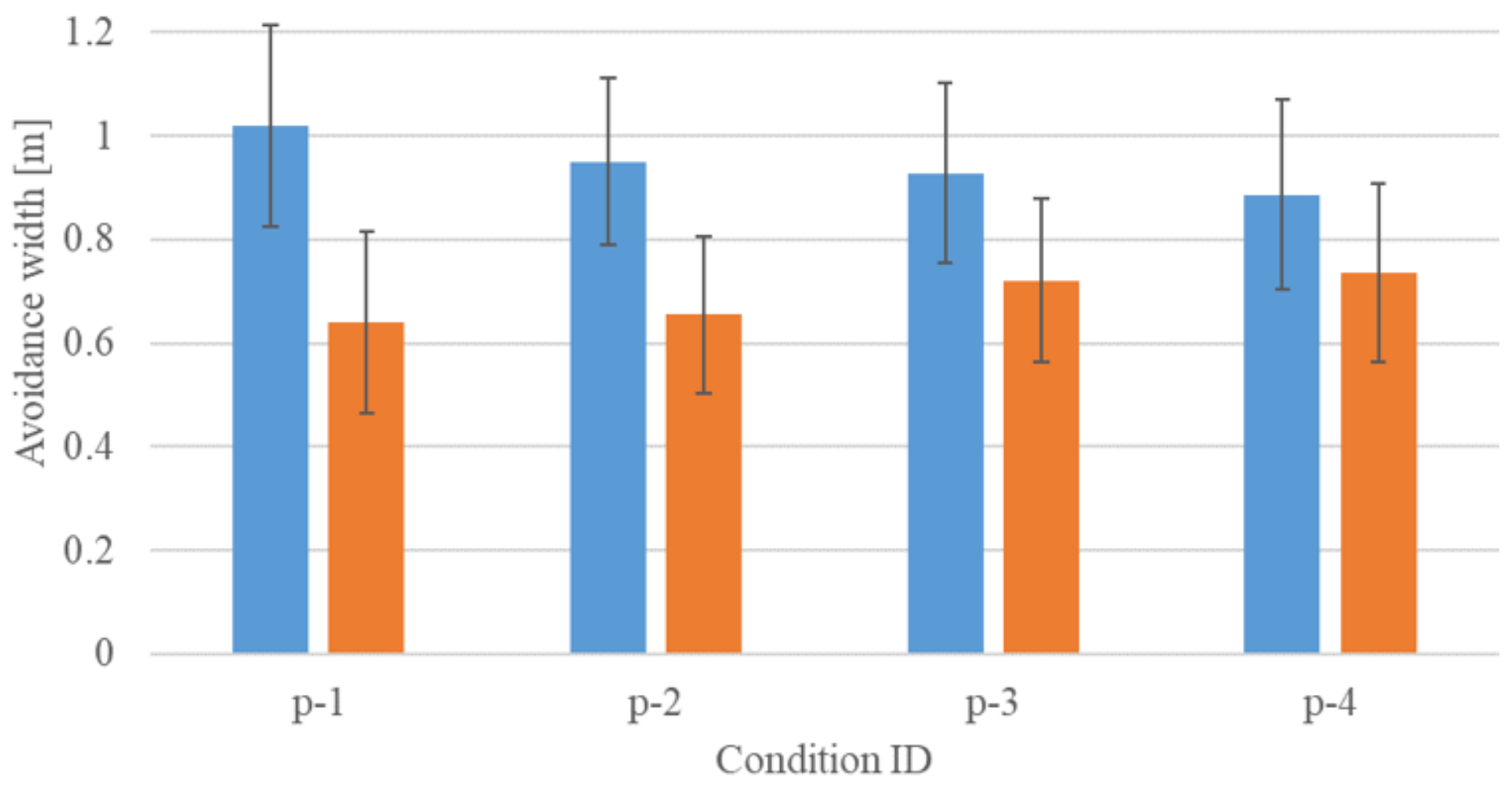

Figure 1

Effect of the PMV's avoidance width on pedestrians' avoidance width 


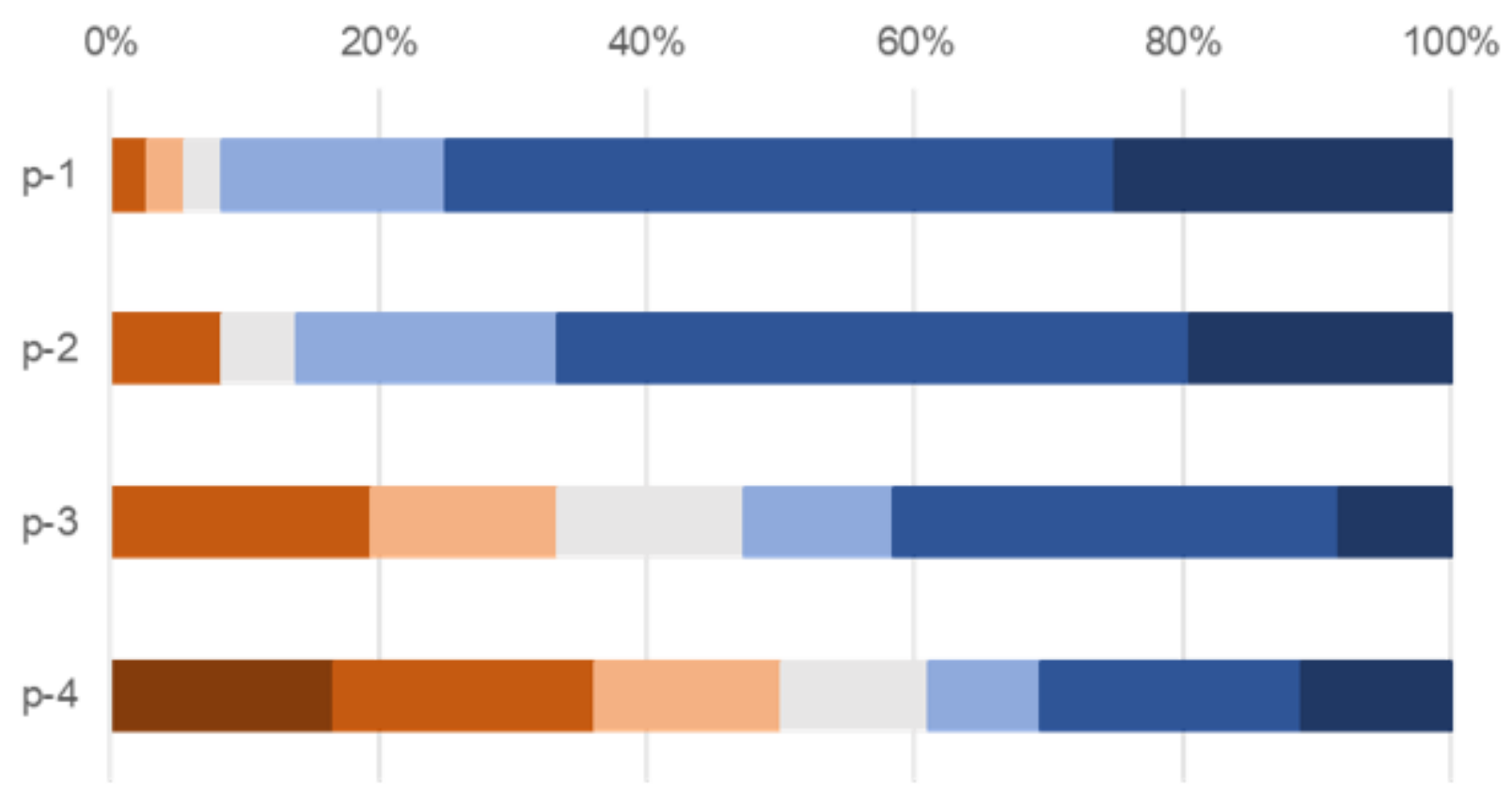

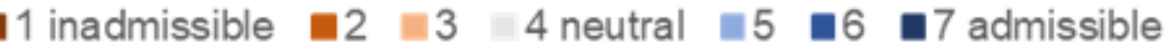

Figure 2

Effect of the PMV's avoidance width on pedestrians' subjective evaluation

$$
\text { - Total width } \quad \text { Pedestrian avoidance width }
$$

1.2

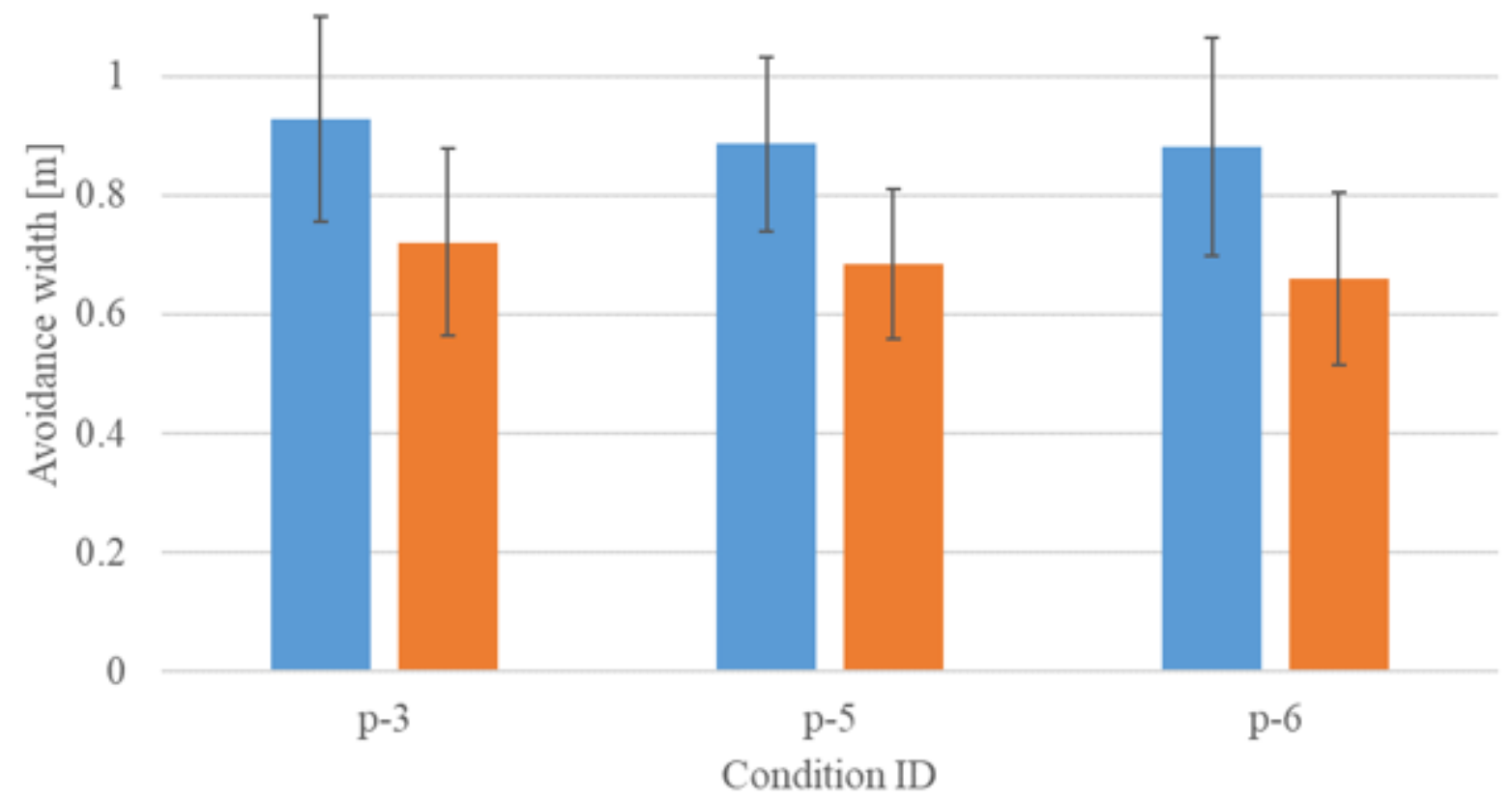

Figure 3 
Effect of the PMV's turning angular velocity on pedestrians' avoidance width

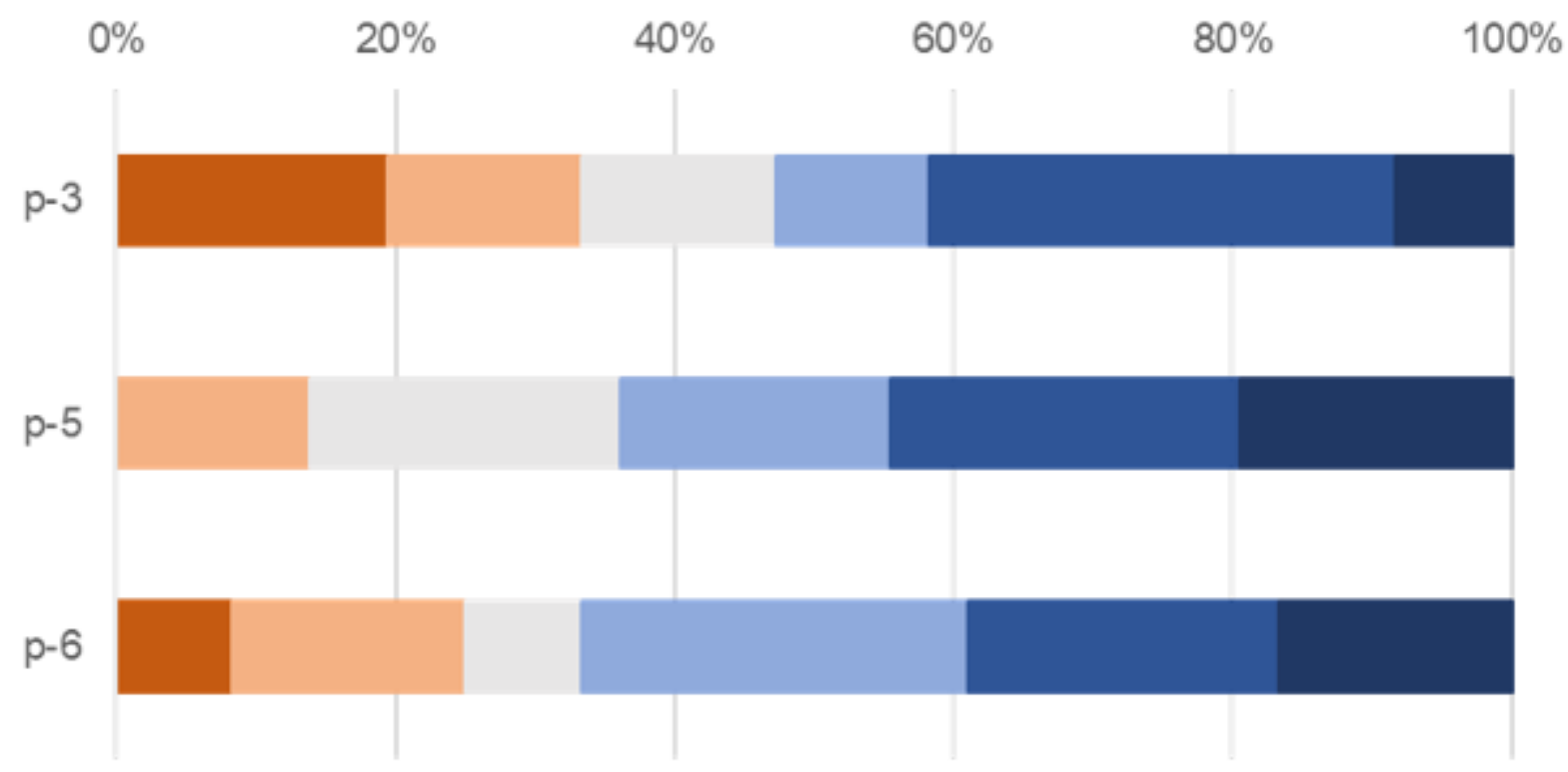

-1 inadmissible $=2 \approx 3 \quad 4$ neutral $=5 \quad 6 \quad$ a 7 admissible

Figure 4

Effect of the PMV's turning angular velocity on pedestrians' subjective evaluation

घMV's avoiding time $\quad$ Pedestan's gazing time against PMV

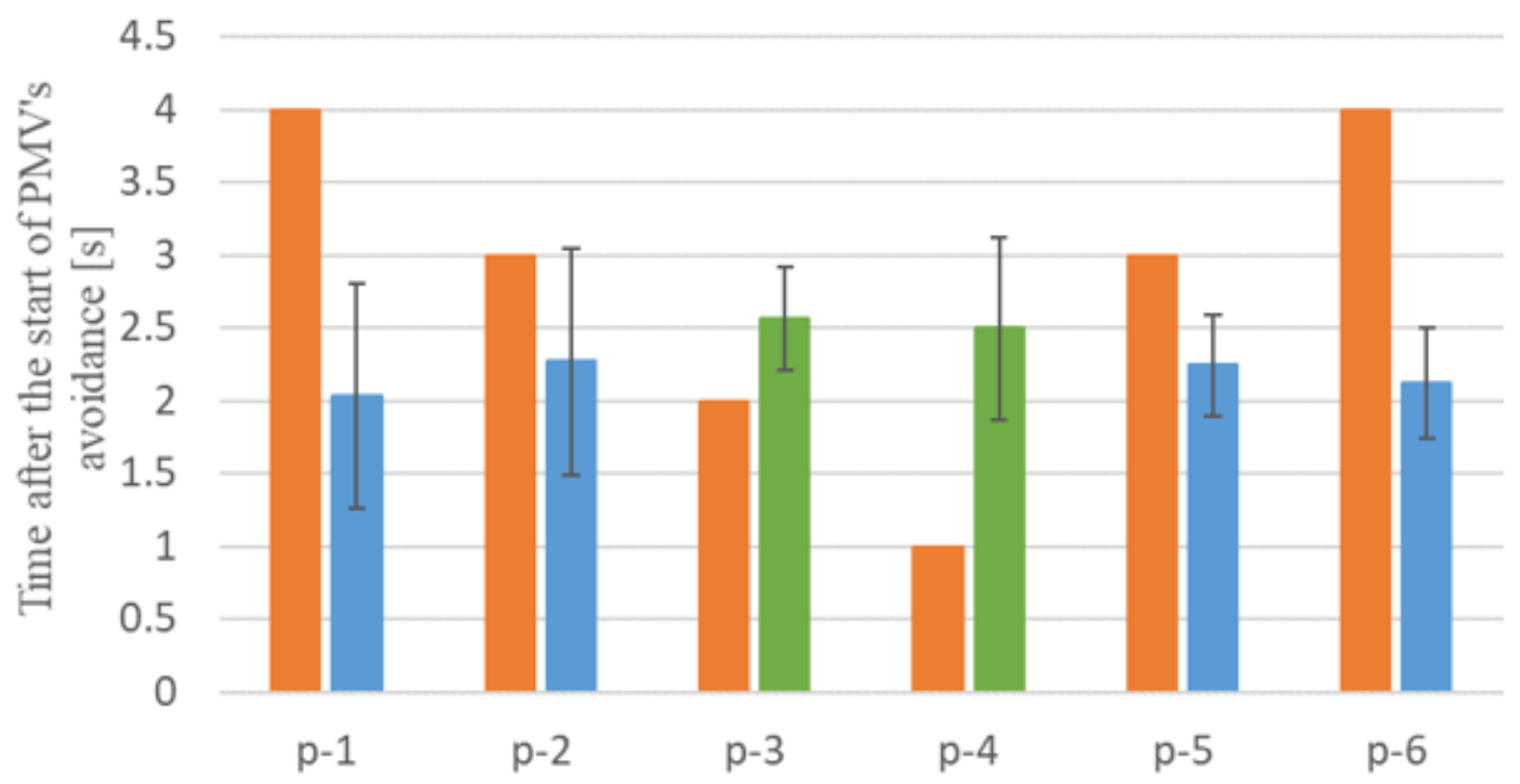

Figure 5 
- Didn't gazed the end of PMV's avoiding behavior

- Gazed the end of PMV's avoiding behavior

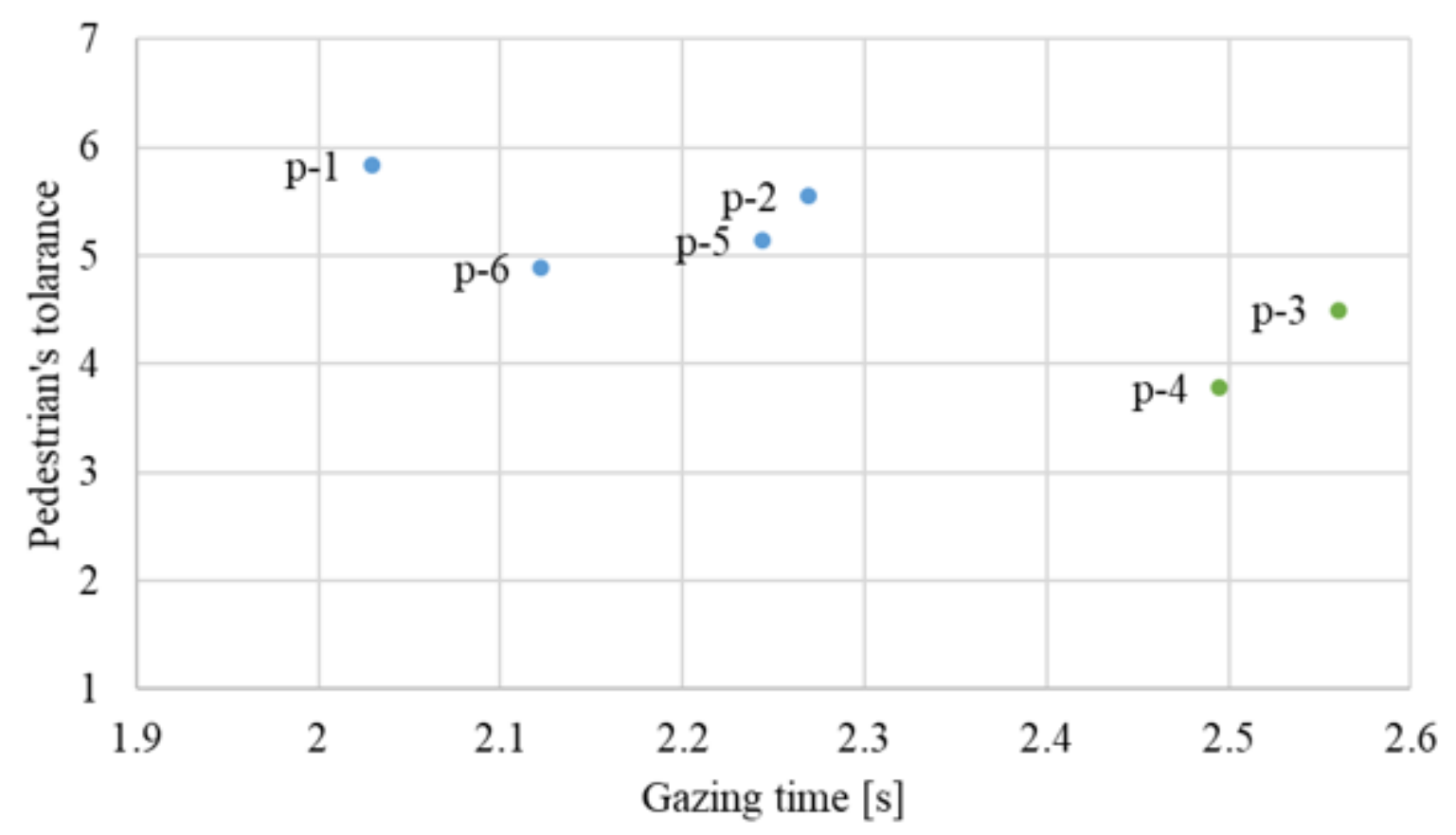

Figure 6

Relationship between pedestrian's tolerance and gazing time against PMV 


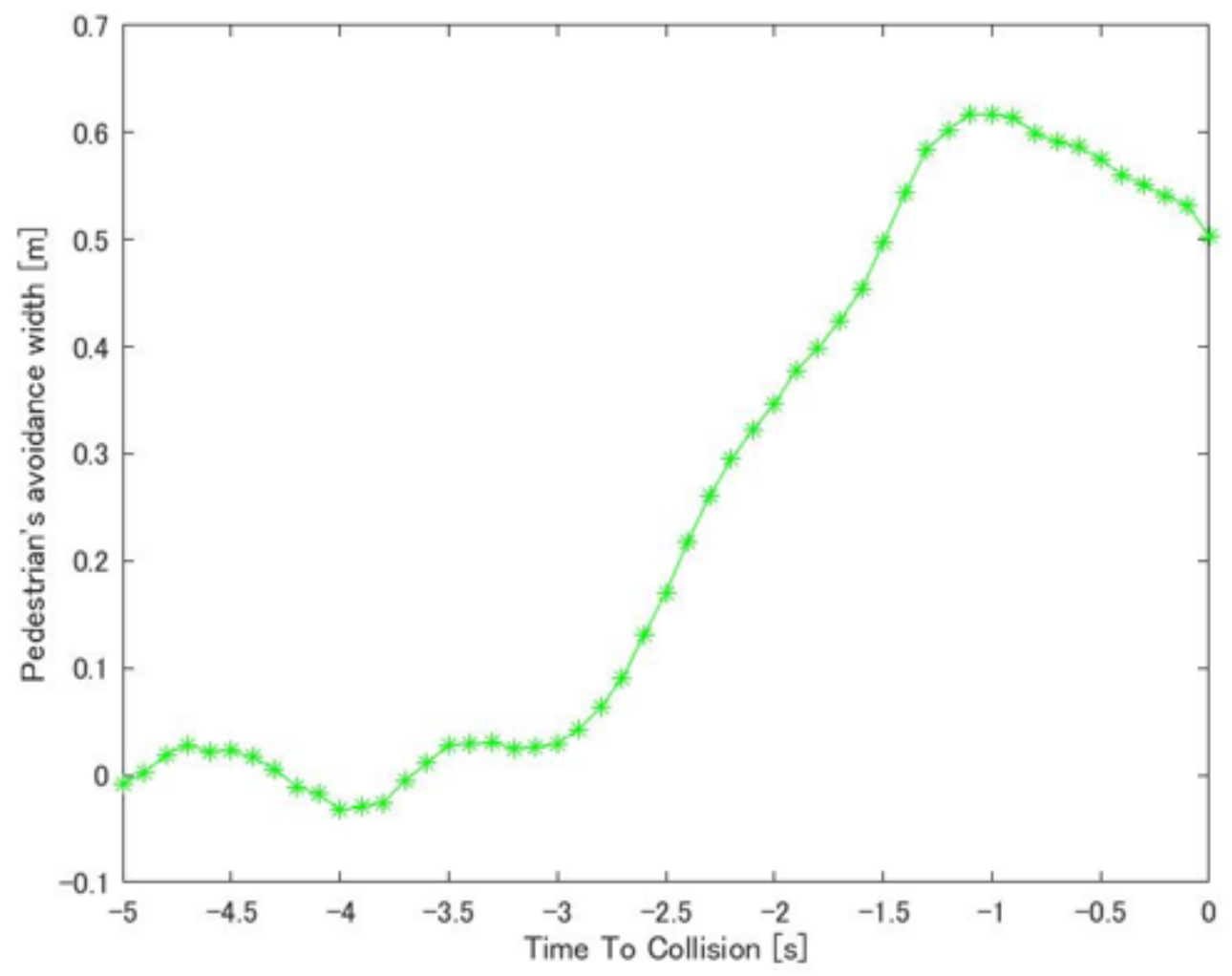

Figure 7

Transition of pedestrians' avoidance maneuver (Participant: G, Condition: p-5)

The horizontal axis represents the time until the moment when the pedestrian passes the PMV. 


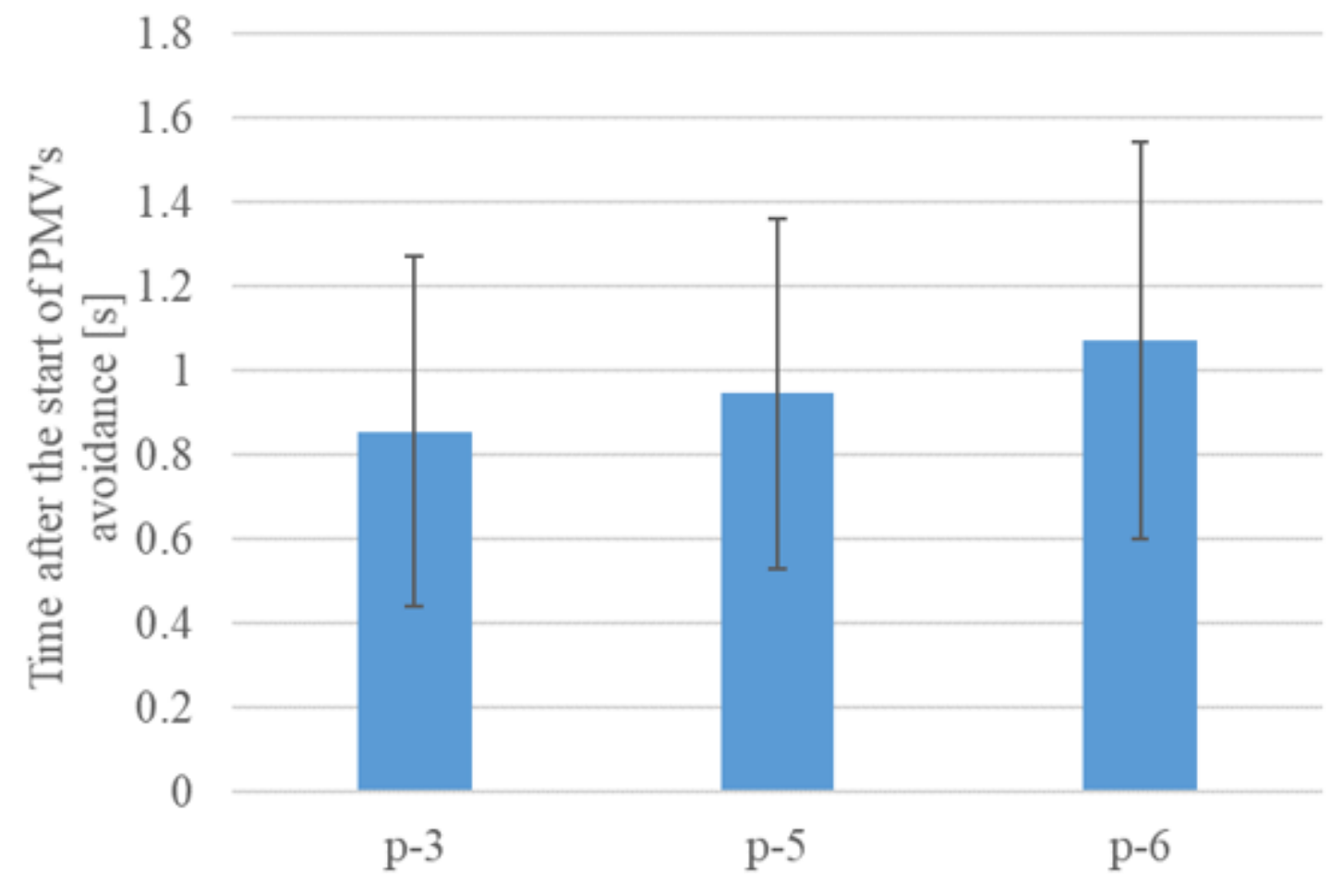

Figure 8

Time difference between the start of the PMV and pedestrian avoidance

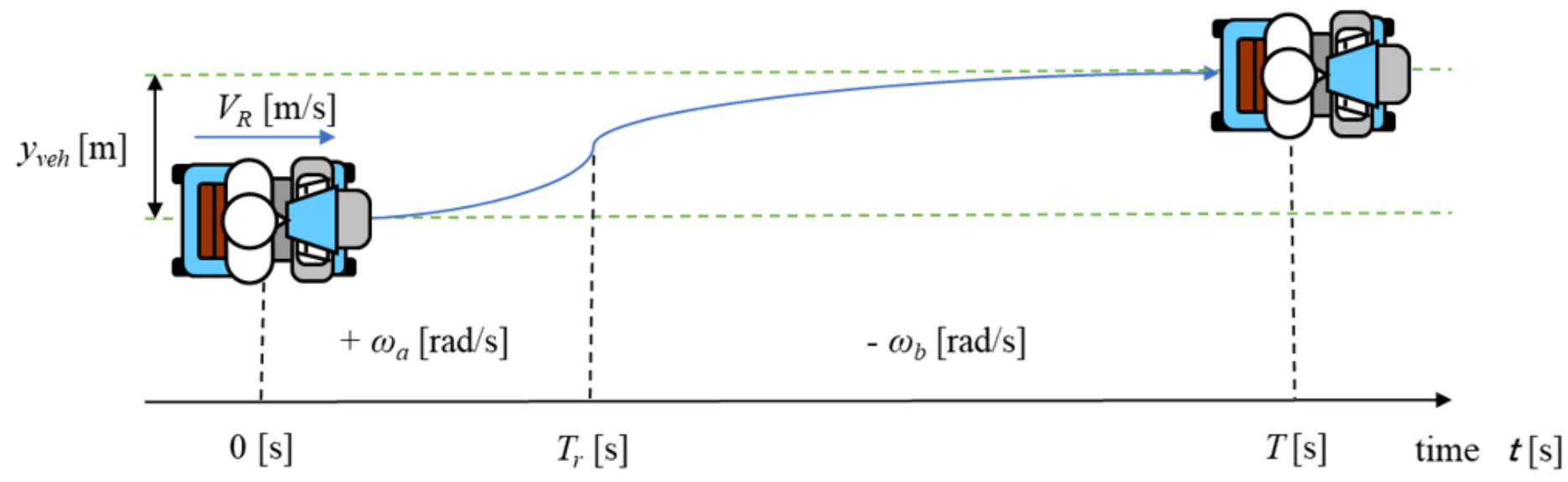

Figure 9

Conceptual diagram of the proposed method 


\section{$\square 2 \mathrm{sec}$ conditions $\square 3 \mathrm{sec}$ conditons}

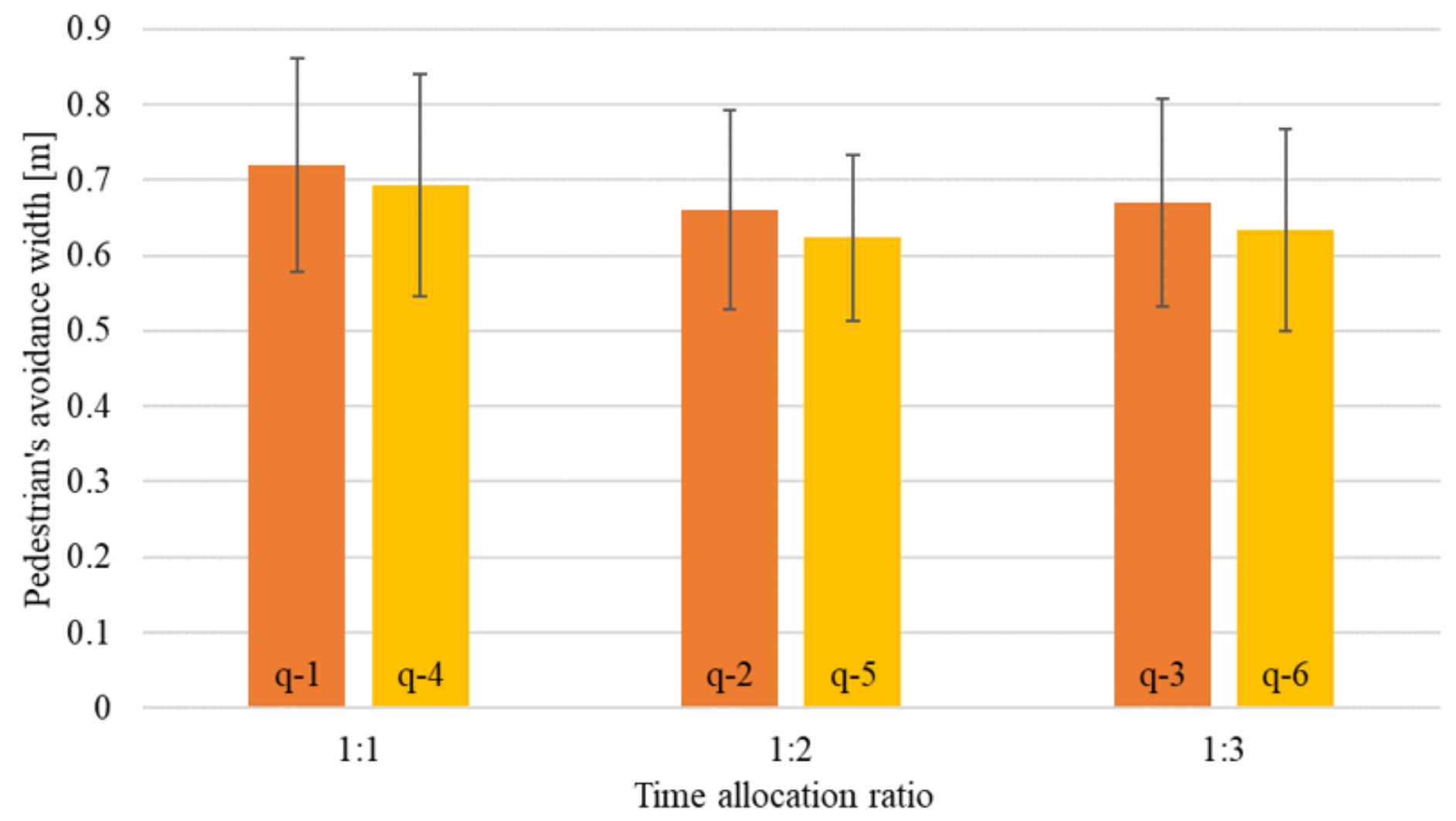

Figure 10

Pedestrians' avoidance width in the evaluation experiment 


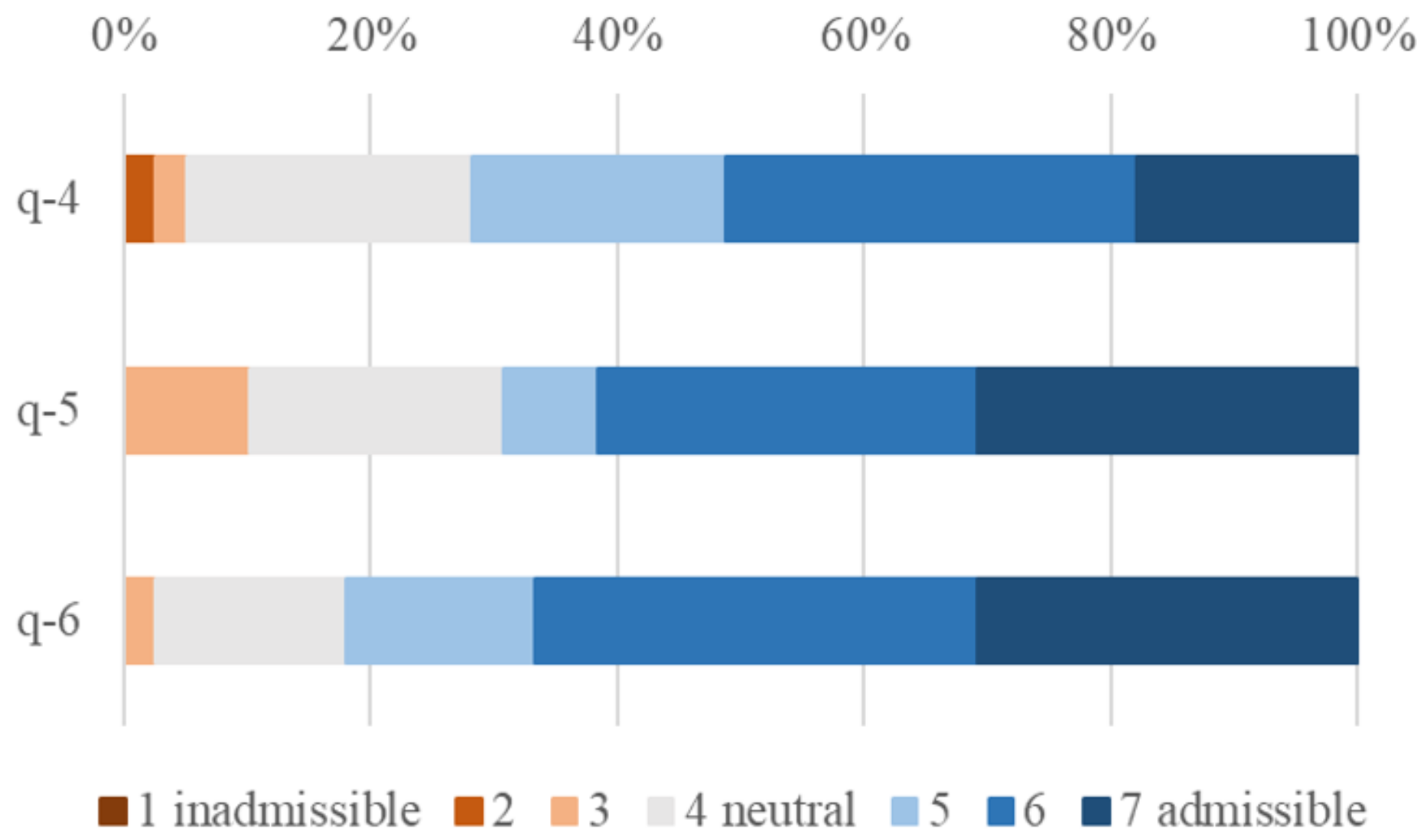

Figure 11

Pedestrians' tolerance evaluation in the evaluation experiment ( 3 s condition) 